\title{
Biomimetic Polyphenol Coatings for Antioxidant Active Packaging Applications
}

3

4 Maxine J. Roman ${ }^{1}$, Eric A. Decker ${ }^{1,2}$, Julie M. Goddard ${ }^{1}$

5

$6{ }^{1}$ Department of Food Science, University of Massachusetts, 102 Holdsworth Way, Amherst, MA

7 01003, USA

$8{ }^{2}$ Bioactive Natural Products Research Group, Department of Biochemistry, Faculty of Science,

9 King Abdulaziz University, P. O. Box 80203 Jeddah 21589 Saudi Arabia

10

11

12

13

14 


\section{ABSTRACT}

16 Oxidative instability of food, pharmaceutical, and consumer products can be promoted by trace

17 metals, especially iron and copper, with subsequent propagation of free radicals. Plant-derived

18 phenolic compounds that contain catechols are reported to have free radical scavenging, metal

19 chelating and surface adhesion properties upon polymerization. The objective of this study was

20 to synthesize biomimetic polyphenol coatings for development of antioxidant active packaging

21 materials. Two synthetic routes were explored to apply polyphenol coatings to the surface of

22 polypropylene by in situ polymerization of a mixture of catechol and catechin and oxidative

23 polymerization with laccase and in alkaline saline. Both polyphenol coatings demonstrated

24 potent metal chelating and radical scavenging capacity, which suggest potential antioxidant

25 capacity. Dual functionality of polyphenol coatings as potent antioxidants and anchors makes

26 them a promising candidate for active packaging coatings that can inhibit metal-promoted

27 oxidative degradation.

\section{KEYWORDS}

30 Catechol; catechin; antioxidant active packaging; metal chelator; radical scavenger; biomaterial 
A major challenge to food, consumer products, and pharmaceutical industries is retaining

33 stability of oxidation-sensitive bioactive compounds, such as unsaturated fatty acids, carotenoids,

34 flavonoids, vitamins, and drugs. Oxidative stability of such compounds is determined by the

35 propagation of free radicals. In dispersions containing bioactive compounds, trace amounts of

36 metals, especially iron and copper, are key prooxidants that contribute to product instability as

37 they promote hydroperoxide decomposition that catalyzes free radical chain reactions [1]. Since

38 it is difficult to fully remove trace metals from raw materials and the processing environment,

39 metal chelators, such as ethylenediaminetetraacetic acid (EDTA), and free radical scavengers,

40 such as butylated hydroxytoluene (BHT), are often added to formulations as antioxidants to

41 stabilize these products. In an effort to reduce use of synthetic additives due to consumer

42 perception of toxicity risk, there has been increasing interest in alternative methods of product

43 preservation using antioxidants obtained from natural sources. Directly substituting natural

44 antioxidants into product formulations is challenging because they tend to be less potent than

45 synthetic additives and therefore must be added in larger amounts that may change a product's

46 organoleptic properties (i.e. color, flavor, viscosity). In order to overcome this challenge,

47 researchers have explored incorporation of natural antioxidants into active packaging coatings.

48 Antioxidant active packaging coatings may be applied to the product contact surface of common

49 packaging materials by non-covalent or covalent attachment of antioxidants that are applied with

50 or without a carrier polymeric resin [2]. These coatings are designed to either scavenge

51 prooxidants from the product or slowly release antioxidants from the packaging material.

52 The majority of research on antioxidant active packaging coatings has been focused on

53 application of free radical scavengers on the surface of packaging materials. Contini, et al. [3]

54 spray deposited citrus extract on plasma pretreated polyethylene terephthalate trays that inhibited

55 oxidative degradation of cooked meats. Garces, et al. [4] patented an antioxidant active varnish

56 for use on packaging materials composed of polymeric resin blended with plant extracts that is 
57 designed to scavenge free radicals in packaging headspace [5]. Arrua, et al. [6] covalently 58 attached a polymer containing caffeic acid to the surface of polypropylene and demonstrated its 59 ability to scavenge free radicals and inhibit degradation of vitamin C in orange juice. Recently, 60 antioxidant active packaging coatings by graft polymerization of metal chelating polymers, such 61 as poly(acrylic acid) and poly(hydroxamic acid), have been developed that exhibit functionality 62 across a broad range of $\mathrm{pH}$ values and viscosity conditions and in the presence of competing ions 63 [7-11]. Metal chelating active packaging coatings extended the lag phase of lipid oxidation in 64 soybean oil-in-water emulsions and demonstrated improved performance for metal chelating 65 polymers with high iron affinity $[12,13]$.

Among free radical scavenging antioxidants derived from natural sources, plant-derived phenolic compounds that contain catechols are reported to have high affinity for iron (e.g. catechol $\log \beta^{\mathrm{Fe}(\mathrm{III})}=43.76$, catechin $\left.\log \beta^{\mathrm{Fe}(\mathrm{III})}=47.4\right)[14,15]$. In addition to their antioxidant capacity, phenolic compounds that contain catechols have been researched as biomimetics of mussel adhesive proteins making them ideal candidates for coating technologies [16-18]. With this in mind, the reported work seeks to design biomimetic catechol-based polyphenol surface

72 coatings for the development of antioxidant active packaging materials that provide dual antioxidant functionality, both by scavenging free radicals directly and by removing trace transition metals from the system.

75

A facile method of coating plant phenols onto material surfaces is through oxidative polymerization. Polymerized plant phenols have been noted to have adhesive properties on many materials relevant to packaging due to their structural similarities to the widely researched mussel adhesive proteins. Jeon, et al. [19] demonstrated that plant phenols can be polymerized onto a variety of material surfaces, including aluminum, glass, polyethylene terephthalate, and polypropylene, with the assistance of the oxidase enzyme, laccase. Barrett, et al. [20] prepared

81 polyphenol coatings from plant phenols by polymerization in an alkaline aqueous buffer, similar 82 to reported methods for the preparation of polydopamine coatings [17]. However, there has yet to 83 be an investigation of the ability of such polyphenol coatings to function as both free radical 
84 scavengers and metal chelators for antioxidant applications. The objective of this study was to 85 synthesize a biomimetic polyphenol coating for development of antioxidant packaging materials with both metal chelating and free radical scavenging character. Polyphenol coatings were

87 applied to the surface of polypropylene by in situ polymerization of a mixture of catechol and catechin $(2.5 \mathrm{mg} / \mathrm{ml}$ catechol; $2.5 \mathrm{mg} / \mathrm{ml}$ catechin $)$ via enzymatic polymerization with laccase (1 $\mathrm{mg} / \mathrm{ml}$ in $100 \mathrm{mM}$ sodium acetate buffer, $\mathrm{pH} 5$ and methanol (9:1)) or oxidative polymerization in alkaline saline (100 mM bicine, $600 \mathrm{mM}$ sodium chloride, $\mathrm{pH}$ 8). Ability of the polyphenol

91 coatings to chelate copper and iron ions and scavenge free radicals was demonstrated. Polymerization of the catechol and catechin by laccase $(\mathrm{pH} 5)$ and in alkaline saline $(\mathrm{pH}$

93 8) was immediately observed by a change in the color of the reaction solution to a dark brown 94 color, characteristic of polyphenol formation. Deposition of the polymerized phenolic coating 95 onto the surface of polypropylene was identified by similar generation of a dark brown color on 96 the polypropylene film after $24 \mathrm{~h}$ in the reaction solution (Figure S1). It is important to note, that 97 for the oxidative polymerization reaction conducted at $\mathrm{pH} 8$ without laccase, preliminary 98 experiments revealed that a minimum of $200 \mathrm{mM}$ sodium chloride was necessary for the 99 polyphenol to be deposited on the surface, with optimum surface deposition at $600 \mathrm{mM}$ sodium 100 chloride (data not shown). Salt ions may shield charges on polyphenols formed under alkaline 101 conditions to allow for better interactions with the hydrophobic polypropylene surface. Previous 102 work in agreement with our findings was conducted by Sileika [21] who found that alkaline 103 saline enabled improved surface deposition of polymerized phenolic compounds compared to 104 pure water. Surface chemistry of the polyphenol coated materials was characterized by ATR-FTIR spectroscopy (Figure 1). Laccase assisted polyphenol coatings exhibited a strong O-H

107 absorbance band (3000-3680 $\left.\mathrm{cm}^{-1}\right)$, two $\mathrm{C}=\mathrm{C}$ absorbance bands $\left(1590,1518 \mathrm{~cm}^{-1}\right)$ attributed to

108 benzene rings on catechol and catechin, and several $\mathrm{C}-\mathrm{O}$ absorbance bands that may be attributed 109 to crosslinks of catechol and catechin or catechol ligands $\left(1050-1290 \mathrm{~cm}^{-1}\right)$. These results are in 110 agreement with reported spectra collected from laccase-assisted polymerization of catechol, 
111 catechin, and their combination [19]. The polyphenol coating produced by alkaline saline

112 polymerization of catechol and catechin exhibited a similar ATR-FTIR spectrum, with slight

113 differences in the intensity and number of absorbance bands for $C=C\left(1606,1575,1537 \mathrm{~cm}^{-1}\right)$

114 and $\mathrm{C}-\mathrm{O}$ bonds $\left(1050-1290 \mathrm{~cm}^{-1}\right)$, which suggests that this polyphenol coating may have a

115 different structure than the polyphenol coating formed by laccase assisted polymerization.

116 Oxidation of a phenol by laccase typically involves loss of a single electron that results in the

117 formation of a cationic radical, whereas alkaline oxidation results in the formation of an anionic

118 radical. Different reactive radicals may influence the structure of the polyphenol formed by

119 subsequent polymerization reactions. Further characterization of polyphenol molecular structure

120 is necessary to confirm such differences as well as hypothesize polymerization reaction pathways

121 and will be the subject of future work. The number of available catechol groups on the surface

122 was assessed by Folin Ciocalteu assay (Figure 1B). Laccase assisted polyphenol coatings

123 contained more than twice the number of available phenolic groups than alkaline saline

124 polyphenol coatings $\left(86.7 \pm 12 \mathrm{nmol}\right.$ catechol eqv. $\mathrm{cm}^{-2}$ and $33.1 \pm 7.3 \mathrm{nmol}$ catechol eqv. $\mathrm{cm}^{-2}$,

125 respectively), while uncoated PP contained an insignificant amount of phenol groups $(0.791 \pm$

$1260.14 \mathrm{nmol}$ catechol eqv. $\left.\mathrm{cm}^{-2}\right)$.

128 Figure 1. (a) Representative ATR-FTIR spectra and (b) total phenol content (n=4) of native 129 PP and polyphenol coated PP.
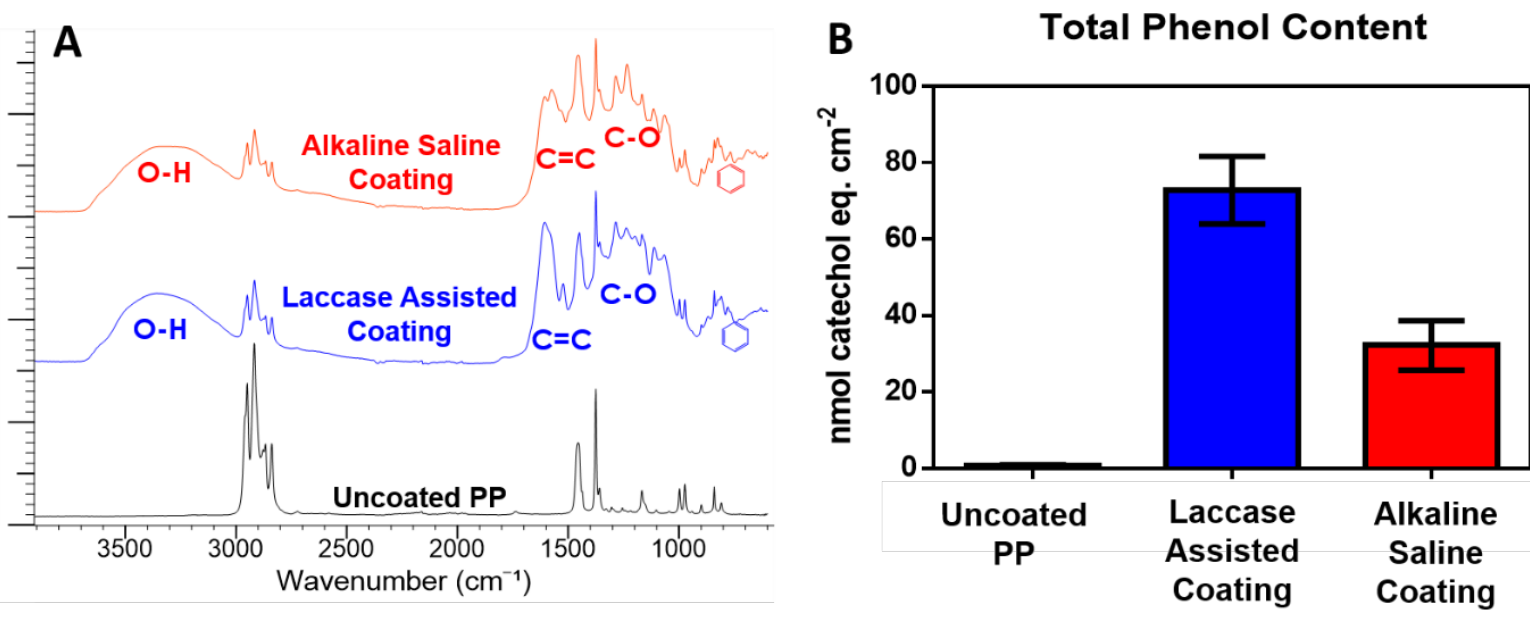
To determine the effect of coating preparation method on surface morphology, surface

133 scanning electron microscopy (SEM) images of coated and uncoated materials were taken

134 (Figure 2). Uncoated PP exhibited a smooth, uniform surface (Figure 2A). SEM micrographs

135 confirmed the deposition of laccase assisted and alkaline saline polyphenol coatings onto the

136 surface of polypropylene films. Laccase assisted polyphenol coatings significantly changed the

137 surface morphology of the polypropylene to a rough surface with aggregates of polyphenols

138 throughout the coating (Figure 2B). The coated surface also exhibited cracks throughout most

139 likely caused by dehydration of the hydrophilic surface prior to imaging. The alkaline saline

140 polyphenol coating was smoother than the laccase assisted polyphenol coating and did not have

141 any visible cracking (Figure 2C). Compared to the uncoated PP material, both polyphenol coated

142 surfaces were rougher with presence of polyphenol aggregates. These observations are consistent

143 with previous research on in situ polymerization of dopamine on glass and aluminum [22], which

144 suggests that the hydrophobic surface properties of polypropylene did not significantly affect

145 coating morphology. Changes in the surface morphology of coated PP materials were evident

146 across the surface, demonstrating uniform application of the polyphenol coatings. Laccase

147 assisted polyphenol coatings were approximately twice the thickness of alkaline polyphenol

148 coatings (laccase assisted coating: $1087 \pm 45.3 \mathrm{~nm}$, alkaline coating: $506.5 \pm 32.1 \mathrm{~nm}$ ). This

149 disparity between coating thickness corresponds with differences in the coatings available

150 phenolic groups (Figure 1B).

152 Figure 2. Representative SEM micrographs of (a) native PP, (b) laccase-assisted polyphenol 153 coated PP, and (c) alkaline saline polyphenol coated PP (10,000x magnification). 


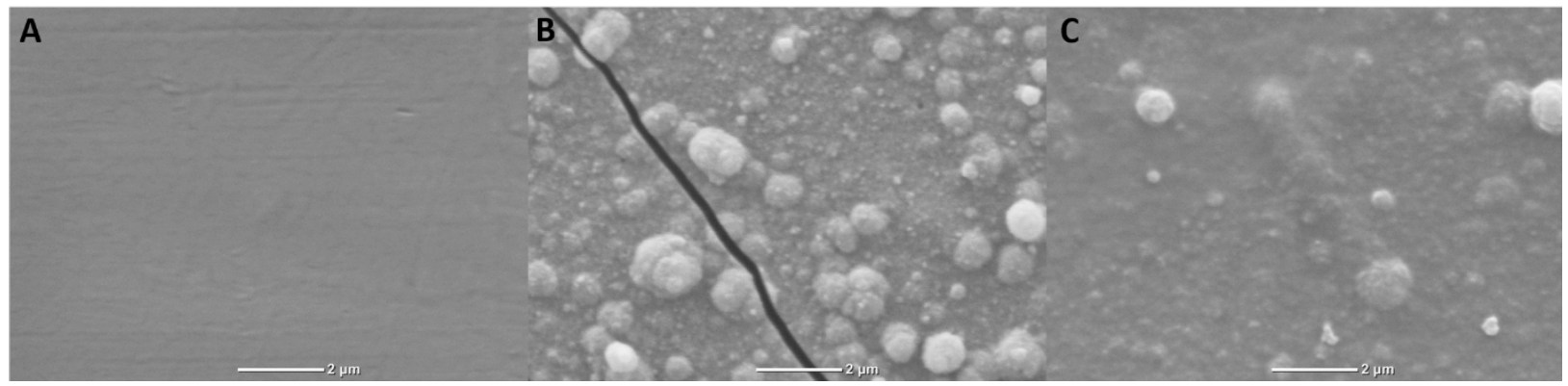

The antioxidant efficacy of the polyphenol coated materials was characterized by

157 screening both metal chelating and free radical scavenging capacity. The ability to chelate

158 ferrous, ferric, and cupric ions was measured at $\mathrm{pH}$ 3-5 to reflect a $\mathrm{pH}$ range of relevant 159 oxidation prone food and consumer products (Figure 3). Polyphenol coatings prepared by both

160 synthetic routes exhibited significant ferrous ion chelating capacity that was $\mathrm{pH}$ dependent.

161 Ferrous chelating capacity decreased with decreasing $\mathrm{pH}$ value, with optimum ferrous ion

162 chelation at $\mathrm{pH}$ 5. In contrast, polyphenol coated materials exhibited optimum ferric ion

163 chelating capacity at $\mathrm{pH}$ 4. The decline of ferric ion chelating capacity at $\mathrm{pH} 5$ may be attributed

164 to loss of ferric ion solubility at increasing $\mathrm{pH}$ values and/or oxidation of catechol hydroxyl

165 groups by ferric ion that would result in the formation of quinones that have a low affinity for 166 iron [23]. Lack of the ability of ferrous ions to convert hydroxyls to quinones could also help 167 explain why chelating capacity of ferrous ions at $\mathrm{pH} 4$ and 5 were higher than ferric ion chelating 168 capacity. There was no significant difference in ferric and ferrous ion chelating capacity at pH 3. 169 Despite significant differences in total phenol content (Figure 1B) and coating thickness, there 170 was no significant difference in iron chelating capacity of the laccase assisted polyphenol coating 171 and alkaline saline polyphenol coating. Similarities in iron chelating capacity suggests that 172 chelating ligands of the polyphenolic coatings may be most active at the surface. Both 173 polyphenol coated materials demonstrated lower copper chelating capacity than iron chelating 174 capacity. Optimum copper chelation was demonstrated at pH 5 and decreased with decreasing $175 \mathrm{pH}$ values, with no significant difference between uncoated material and polyphenol coated material at $\mathrm{pH}$ 3. Similar to ferric ion, cupric ion may oxidize the catechol ligands to hinder 
177 effective chelation of metal ions. Ability to chelate ferrous ion, ferric ion, and cupric ion suggests

178 that the reported polyphenol coated films may inhibit oxidative degradation reactions.

179 Figure 3. (a) Ferrous ion, (b) ferric ion, and (c) cupric ion chelating capacity (n=4) of native 180 PP and polyphenol coated PP at different $\mathbf{p H}$ values (3.0, 4.0, and 5.0).
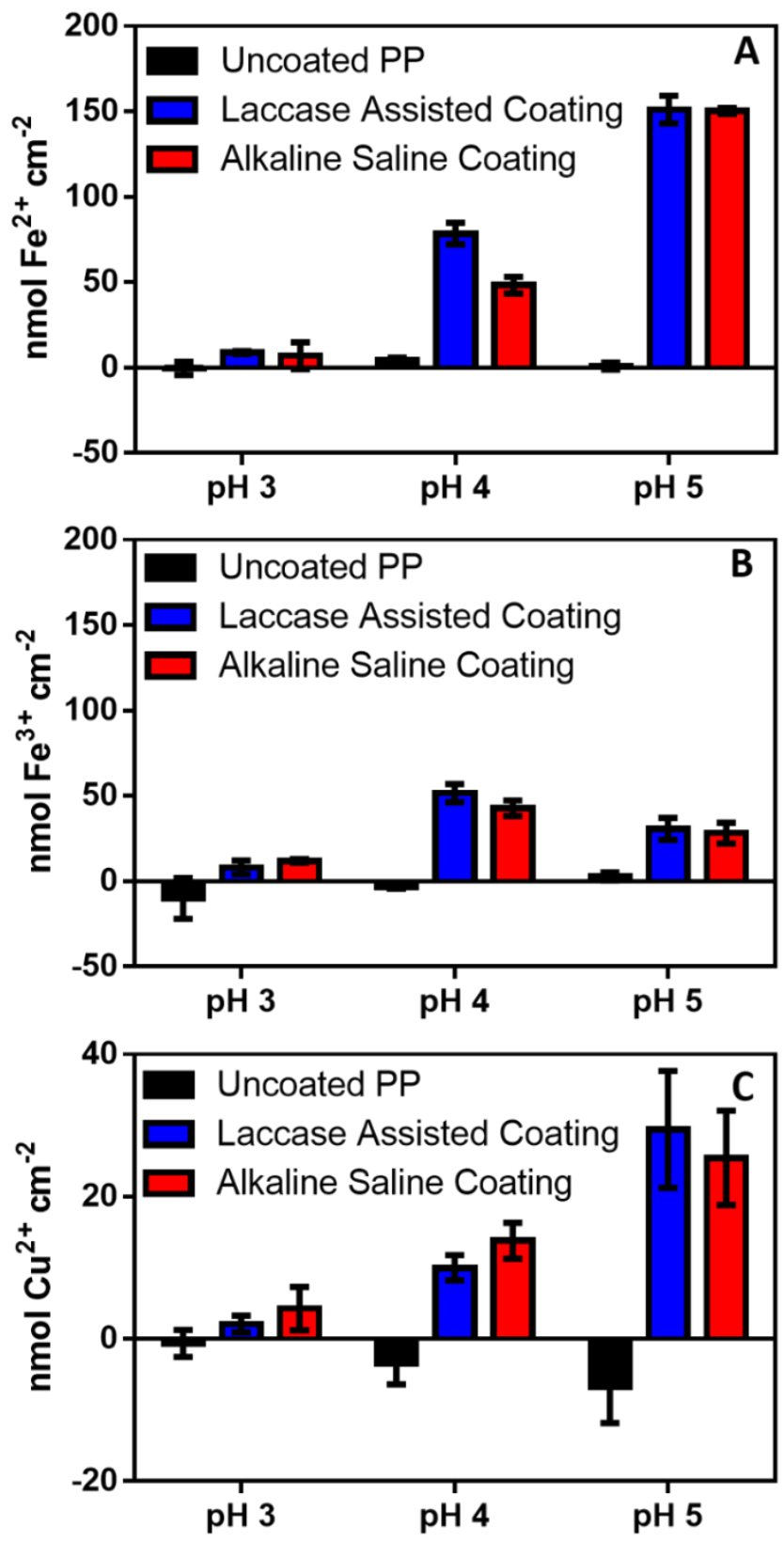

Free radical scavenging capacity of polyphenol coated films was assessed by oxygen

183 radical absorbance capacity (ORAC, Figure 4). For this assay, the efficacy of an antioxidant is 
184 quantified by its ability to protect fluorescein from oxidative degradation by scavenging peroxyl

185 radicals generated by AAPH [24]. Polyphenol coated films were able to decrease the overall rate

186 of fluorescein degradation, which is indicative of radical scavenging capacity. Based on area

187 under the curve, laccase assisted polyphenol coating was estimated to have $22.51 \mathrm{nmol}$ Trolox eq

$188 \mathrm{~cm}^{-2}$ and alkaline saline polyphenol coating was estimated to have $22.62 \mathrm{nmol}$ Trolox eq $\mathrm{cm}^{-2}$. As

189 observed in the metal chelating capacity measurements, total phenol content did not appear to

190 influence the free radical scavenging capacity of the coated films. Compared to the Trolox

191 positive control, which demonstrates free radical scavenging behavior typical of soluble

192 antioxidants, neither polyphenol coated materials exhibited a distinct lag phase for the induction

193 of fluorescein oxidation (Figure 4). The lack of lag phase may be due to diffusion limitations of

194 surface immobilized antioxidants to quench peroxyl radicals or possible higher redox potential of

195 surface immobilized polyphenols compared to Trolox $\left(\mathrm{E}^{\circ}=480 \mathrm{mV}\right)$ [24]. Nevertheless, both

196 coatings demonstrated potent free radical scavenging capacity, which may contribute to their

197 overall antioxidant capacity when applied to packaging materials.

198 Figure 4. (a) Radical scavenging capacity of polyphenol coated PP $(n=4)$ demonstrated by 199 delayed decay of fluorescent probe in oxygen radical scavenging capacity (ORAC) assay. 200 (b) Approximate radical scavenging capacity (Trolox eqv.) estimated by area under the 201 curve.

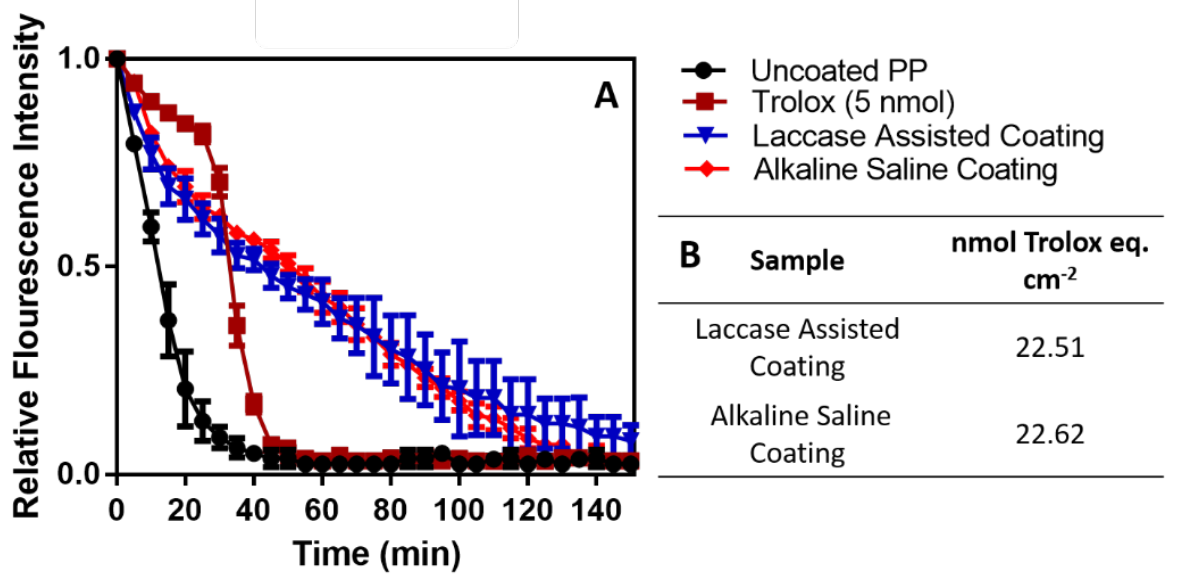

203 Phenolic compounds that are derived from plants represent a promising source for

204 antioxidants that may be used to hinder degradation of oxidation-sensitive bioactive compounds. 
205 Application of such phenolic compounds in active packaging coatings may prevent their

206 interference with the organoleptic properties of food and consumer products, while still

207 delivering antioxidant protection of bioactive compounds. Recent research has demonstrated that

208 compounds that bear structural similarities to mussel adhesive proteins, such as catechol-based

209 plant phenols, have functional adhesive properties useful for the development of coating

210 technologies. This proof of principle study demonstrated that polyphenol coatings can be applied

211 by in situ laccase assisted polymerization or alkaline saline polymerization of catechol and

212 catechin possess dual antioxidant capacity, exhibiting both metal chelating and radical

213 scavenging properties. These findings warrant further investigation for the development of

214 antioxidant polyphenol coatings for active packaging applications in food, consumer products,

215 and pharmaceutical industries.

\section{ACKNOWLEDGEMENTS}

218 We would like to acknowledge the Peter Salmon Graduate Fellowship (Dept of Food Science,

219 UMass Amherst) and Northeast Alliance for Graduate Education and the Professoriate

220 Fellowship for their support. This project was supported by Agriculture and Food Research

221 Initiative Grant no. 2012-67017-30157 from the USDA National Institute of Food and

222 Agriculture, Improved Processing Technologies (A1351). 


\section{References}

225

1. Waraho, T.; McClements, D.J.; Decker, E.A. Mechanisms of lipid oxidation in food dispersions. Trends Food Sci. Technol. 2011, 22, 3-13.

2. Bastarrachea, L.J.; Wong, D.E.; Roman, M.J.; Lin, Z.; Goddard, J.M. Active packaging coatings. Coatings 2015, 5, 771-791.

3. Contini, C.; Katsikogianni, M.; O’Neill, F.; O’Sullivan, M.; Boland, F.; Dowling, D.; Monahan, F. Storage stability of an antioxidant active packaging coated with citrus extract following a plasma jet pretreatment. Food Bioprocess Tech. 2014, 7, 2228-2240.

4. Garces, O.; Nerin, C.; Beltran, J.; Roncales, P. Antioxidant active varnish. European Patent EP1477159-Al 2003.

5. Pezo, D.; Salafranca, J.; Nerín, C. Determination of the antioxidant capacity of active food packagings by in situ gas-phase hydroxyl radical generation and high-performance liquid chromatography-fluorescence detection. J. Chromatogr. A 2008, 1178, 126-133.

6. Arrua, D.; Strumia, M.C.; Nazareno, M.A. Immobilization of caffeic acid on a polypropylene film: Synthesis and antioxidant properties. J. Agric. Food Chem. 2010, 58, 9228-9234.

7. Tian, F.; Roman, M.J.; Decker, E.A.; Goddard, J.M. Biomimetic design of chelating interfaces. J. Appl. Polym. Sci. 2015, 132, 41231.

8. Roman, M.J.; Tian, F.; Decker, E.A.; Goddard, J.M. Iron chelating polypropylene films: Manipulating photoinitiated graft polymerization to tailor chelating activity. J. Appl. Polym. Sci. 2014, 131, 39948.

9. Tian, F.; Decker, E.A.; McClements, D.J.; Goddard, J.M. Influence of non-migratory metalchelating active packaging film on food quality: Impact on physical and chemical stability of emulsions. Food Chem. 2014, 151, 257-265.

10. Ogiwara, Y.; Roman, M.J.; Decker, E.A.; Goddard, J.M. Iron chelating active packaging: Influence of counter-ions and ph value on effectiveness of soluble and immobilized hydroxamate chelators Food Chem. 2015, 196, 842-847.

11. Roman, M.J.; Decker, E.A.; Goddard, J.M. Performance of nonmigratory iron chelating active packaging materials in viscous model food systems. J. Food Sci. 2015, 80, 1965-1973.

12. Tian, F.; Decker, E.A.; Goddard, J.M. Control of lipid oxidation by nonmigratory active packaging films prepared by photoinitiated graft polymerization. J. Agric. Food Chem. 2012, 60, 7710-7718.

13. Tian, F.; Decker, E.A.; Goddard, J.M. Controlling lipid oxidation via a biomimetic iron chelating active packaging material. J. Agric. Food Chem. 2013, 61, 12397-12404. 
14. Avdeef, A.; Sofen, S.R.; Bregante, T.L.; Raymond, K.N. Coordination chemistry of microbial iron transport compounds. 9. Stability constants for catechol models of enterobactin. J. Am. Chem. Soc. 1978, 100, 5362-5370.

15. Elhabiri, M.; Carrër, C.; Marmolle, F.; Traboulsi, H. Complexation of iron (iii) by catecholate-type polyphenols. Inorganica Chimica Acta 2007, 360, 353-359.

16. Waite, J.H. Surface chemistry: Mussel power. Nat. Mater. 2008, 7, 8-9.

17. Lee, H.; Dellatore, S.M.; Miller, W.M.; Messersmith, P.B. Mussel-inspired surface chemistry for multifunctional coatings. Science 2007, 318, 426-430.

18. Sileika, T.S.; Barrett, D.G.; Zhang, R.; Lau, K.H.A.; Messersmith, P.B. Colorless multifunctional coatings inspired by polyphenols found in tea, chocolate, and wine. Angewandte Chemie International Edition 2013, 52, 10766-10770.

19. Jeon, J.-R.; Kim, J.-H.; Chang, Y.-S. Enzymatic polymerization of plant-derived phenols for material-independent and multifunctional coating. J. Mater. Chem. B 2013, 1, 6501-6509.

20. Barrett, D.G.; Sileika, T.S.; Messersmith, P.B. Molecular diversity in phenolic and polyphenolic precursors of tannin-inspired nanocoatings. Chem. Commun. 2014, 50, 7265 7268.

21. Sileika, T.S. Functional biomaterials inspired by natural polyphenols. NORTHWESTERN UNIVERSITY, 2014.

22. He, L.; So, V.L.L.; Xin, J.H. Dopamine polymerization-induced surface colouration of various materials. RSC Advances 2014, 4, 20317-20322.

23. Nkhili, E.; Loonis, M.; Mihai, S.; El Hajji, H.; Dangles, O. Reactivity of food phenols with iron and copper ions: Binding, dioxygen activation and oxidation mechanisms. Food Funct. 2014, 5, 1186-1202.

24. Bisby, R.H.; Brooke, R.; Navaratnam, S. Effect of antioxidant oxidation potential in the oxygen radical absorption capacity (orac) assay. Food Chem. 2008, 108, 1002-1007. 


\section{GRAPHICAL ABSTRACT}
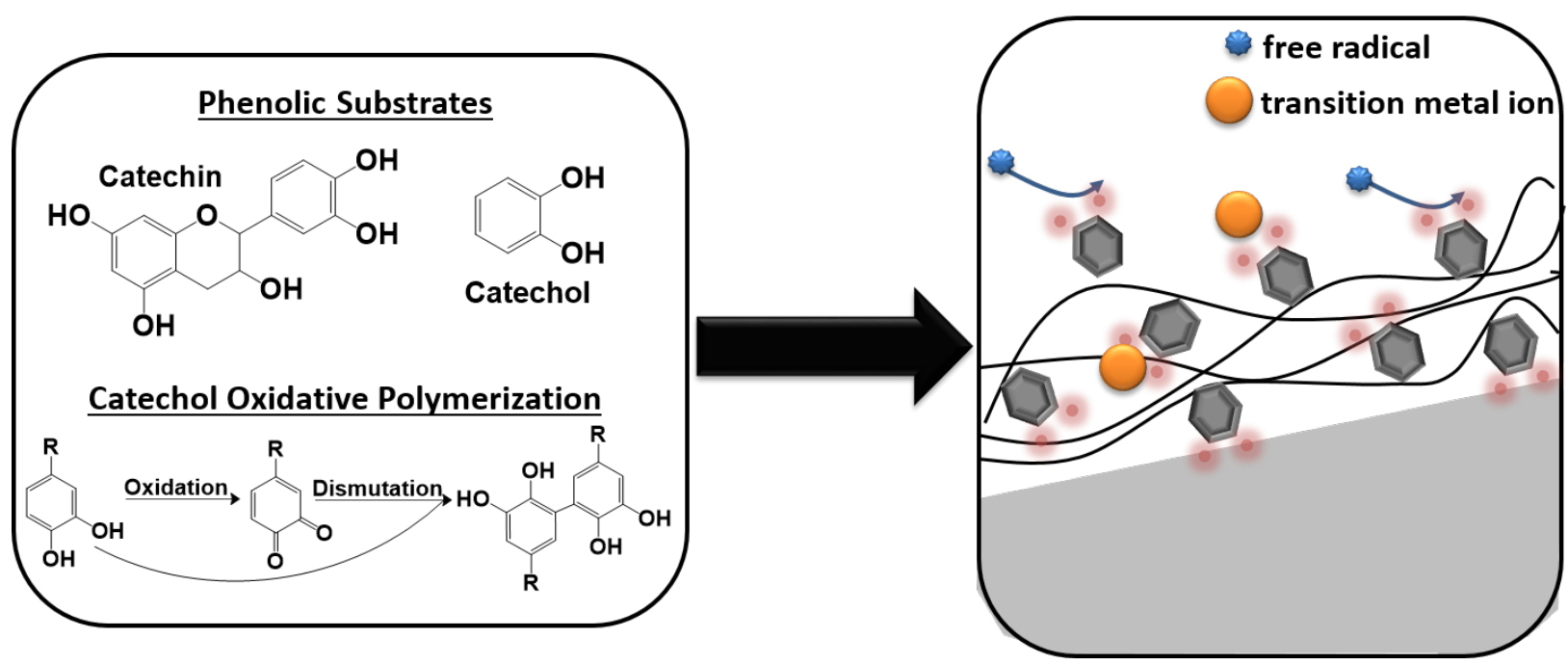